MMCA-97 Conference, pp. 152-159

R. Čiegis (Ed)

(C) 1997 Vilniaus Gedimino technikos universitetas

\title{
SOME ESTIMATES FOR A SPECIAL LINEAR DIFFERENCE PARABOLIC EQUATION
}

\author{
ARTŪRAS ŠTIKONAS \\ Institute of Mathematics and Informatics \\ Akademijos 4, LT-2600 Vilnius, Lithuania \\ e-mail:arturass@ktl.mii.lt
}

\section{ABSTRACT}

The finite-difference scheme for a special linear parabolic equation is investigated. A priori estimates for such finite-difference scheme are derived in the difference analogues of norm on Banach function spaces $V_{2}$ and $W_{2}^{2,1}$.

\section{INTRODUCTION}

A.A. Amosov and A.A. Zlotnik $[2,3]$ obtained some results on the following linear difference scheme $(\mathrm{LDS}, k=0,1)$ :

$$
\bar{\partial}_{t}(\alpha V)=\delta(\kappa \delta V)+\Phi,\left.\delta^{k} V\right|_{i=0, n}=0,\left.V\right|^{j=0}=V^{0} .
$$

The unknown function $V$ is defined on the grid $\bar{\omega}^{h} \times \bar{\omega}^{\tau}$ when $k=0$ and $V$ is defined on the grid $\bar{\omega}_{1 / 2}^{h} \times \bar{\omega}^{\tau}$ when $k=1$. We suppose that $\left.\delta^{k} V^{0}\right|_{i=0, n}=0$. Eq. (1) is defined on the grid $\omega^{h} \times \omega^{\tau}$ when $k=0$ and on the grid $\omega_{1 / 2}^{h} \times \omega^{\tau}$ when $k=1$. So, the grids on which functions $\alpha, \kappa$, and $\Phi$ are defined are known. Let $\Phi=\bar{\partial}_{t} \Phi_{a}+\Phi_{b}+\delta \Phi_{c}$, where $\left.\Phi_{c}\right|_{i=0, n}=0$ when $k=1$.

Lemma 1.1. Let $N^{-1} \leq \alpha, \kappa$, and $q_{l}, r_{l} \in[1, \infty], l=1,2,3,4$; and $\left(2 q_{1}\right)^{-1}+r_{1}^{-1} \leq 5 / 4,\left(2 q_{l}\right)^{-1}+r_{l}^{-1}<1, l=2,3,4$. The next statements are valid:

a) If $\left\|\bar{\partial}_{t} \kappa\right\|_{\infty, 1} \leq N\left(\kappa^{0}=\kappa^{1}\right)$ then

$$
\begin{aligned}
& \|V\|_{Q}+\left\|I_{\tau} \delta V\right\|_{2, \infty} \\
& \quad \leq K(N)\left(\left\|\alpha^{0} V^{0}-\Phi_{a}^{0}\right\|^{(-1) ; k}+\left\|\Phi_{a}\right\|_{Q}+\left\|\Phi_{b}\right\|_{1,1}+\left\|\Phi_{c}\right\|_{2,1}\right),
\end{aligned}
$$


where

$$
\|V\|^{(-1) ; 0}=\sup _{W: W_{0}=W_{n}=0} \frac{(V, W)_{\omega^{h}}}{\|W\|^{(1)}}, \quad\|V\|^{(-1) ; 1}=\sup _{W} \frac{(V, W)_{\omega_{1 / 2}^{h}}}{\|W\|^{(1)}}
$$

b) If $\left\|\alpha^{0}\right\|_{\infty} \leq N$, $\left\|\bar{\partial}_{t} \alpha\right\|_{q_{2}, r_{2}} \leq N$ (or $\left.\left\|\bar{\partial}_{t} \alpha\right\|_{\infty, 1} \leq N\right)$ and $\tau_{\max } \leq \tau^{0}(N)$ or if $\left\|\alpha^{0}\right\|_{\infty} \leq N, \bar{\partial}_{t} \alpha \geq 0$ then

$$
\begin{aligned}
& \left\|\sqrt{\tau} \bar{\partial}_{t} V\right\|_{Q}^{2}+\|V\|_{V_{2}} \leq \\
& \quad \leq K(N)\left(\left\|V^{0}\right\|+\left\|\Phi_{a}^{0}\right\|+\left\|\tau^{-1 / 2} \Phi_{a}\right\|_{Q}+\left\|\Phi_{b}\right\|_{q_{1}, r_{1}}+\left\|\Phi_{c}\right\|_{Q}\right)
\end{aligned}
$$

c) If conditions b) are valid and $\Phi_{a}=0$ then

$$
\|V\|_{C} \leq K(N)\left(\left\|V^{0}\right\|_{\infty}+\left\|\Phi_{b}\right\|_{q_{3}, r_{3}}+\left\|\Phi_{c}\right\|_{2 q_{4}, 2 r_{4}}\right)
$$

d) If $\alpha, \kappa \leq N,\left\|\bar{\partial}_{t} \alpha\right\|_{Q} \leq N,\|\delta \kappa\|_{2, \infty} \leq N$ then

$$
\|V\|^{(2,1)} \leq K(N)\left(\left\|V^{0}\right\|^{(1)}+\|\Phi\|_{Q}+\|V\|_{V_{2}}\right)
$$

\section{NOTATION}

The notation and conventions adopted here are the same as that introduced by A.A. Amosov and A.A. Zlotnik [3]. In the domain $\bar{Q}$ we introduce grids $\overline{\bar{\omega}}^{h}=\left\{\widetilde{q}_{-1} \leq 0=\widetilde{q}_{0}<\widetilde{q}_{1}<\cdots<\widetilde{q}_{n}=L \leq \widetilde{q}_{n+1}\right\}, \bar{\omega}^{h}=\overline{\bar{\omega}}^{h} \backslash\left\{\widetilde{q}_{-1}, \widetilde{q}_{n+1}\right\}$, $\widetilde{\omega}^{h}=\bar{\omega}^{h} \backslash\left\{\widetilde{q}_{0}\right\}, \omega^{h}=\bar{\omega}^{h} \backslash\left\{\widetilde{q}_{0}, \widetilde{q}_{n}\right\}$ with stepsizes $h_{i}=\widetilde{q}_{i}-\widetilde{q}_{i-1}, 1 \leq i \leq$ $n, h_{0}=h_{n+1}=0$, the grids $\bar{\omega}_{1 / 2}^{h}=\left\{\widetilde{q}_{i+1 / 2} \mid \widetilde{q}_{i+1 / 2}=\left(\widetilde{q}_{i}+\widetilde{q}_{i+1}\right) / 2,-1 \leq\right.$ $i \leq n\}, \widetilde{\omega}_{1 / 2}^{h}=\bar{\omega}_{1 / 2}^{h} \backslash\left\{\widetilde{q}_{-1 / 2}\right\}, \omega_{1 / 2}^{h}=\bar{\omega}_{1 / 2}^{h} \backslash\left\{\widetilde{q}_{-1 / 2}, \widetilde{q}_{n+1 / 2}\right\}$ with stepsizes $h_{i+1 / 2}=\left(h_{i}+h_{i+1}\right) / 2,0 \leq i \leq n$, and $\bar{\omega}^{\tau}=\left\{t_{j} \mid 0=t_{0}<t_{1}, \cdots, t_{\bar{n}}=T\right\}$, $\omega^{\tau}=\bar{\omega}^{\tau} \backslash\left\{t_{0}\right\}, \quad \stackrel{\circ}{\omega}^{\tau}=\omega^{\tau} \backslash\left\{t_{1}\right\}$ with stepsizes $\tau_{j}=t_{j}-t_{j-1}, \quad 0<j \leq \bar{n}$. We assume $h_{\max }=\max _{1 \leq i \leq n} h_{i}, \quad \tau_{\max }=\max _{1 \leq j \leq \bar{n}} \tau_{j}, \tau_{0}=0$. Let the grid $\overline{\bar{\omega}} h$ be a quasiuniform, i.e. $N^{-1} \leq h_{i+1} / h_{i} \leq N(0<i<n)$. We consider grid functions $Z=Z^{j}=Z_{i}=Z_{i}^{j}=Z\left(\widetilde{q}_{i}, t_{j}\right), Z=Z^{j}=Z_{i+1 / 2}=Z_{i+1 / 2}^{j}=$ $Z\left(\widetilde{q}_{i+1 / 2}, t_{j}\right)$, and denote $Z \in \mathbb{R}\left(\bar{\omega}^{h} \times \bar{\omega}^{\tau}\right)$ and $Z \in \mathbb{R}\left(\bar{\omega}_{1 / 2}^{h} \times \bar{\omega}^{\tau}\right)$, respectively. For functions $Z_{k}, k=1,2, \ldots$ we use the notation $Z_{k}=Z_{k ; i}$ or $Z_{k}=Z_{k ; i+1 / 2}$.

Let the grid functions $U \in \mathbb{R}\left(\bar{\omega}^{h}\right), \widetilde{U} \in \mathbb{R}\left(\widetilde{\omega}^{h}\right), \stackrel{\circ}{U} \in \mathbb{R}\left(\omega^{h}\right), \bar{V} \in \mathbb{R}\left(\bar{\omega}_{1 / 2}^{h}\right)$, $\widetilde{V} \in \boldsymbol{R}\left(\widetilde{\omega}_{1 / 2}^{h}\right), V \in \boldsymbol{R}\left(\omega_{1 / 2}^{h}\right), Y \in \mathbb{R}\left(\bar{\omega}^{\tau}\right), \stackrel{\circ}{Y} \in \mathbb{R}\left(\omega^{\tau}\right)$ be determined on the grids $\bar{\omega}^{h}, \widetilde{\omega}^{h}, \omega^{h}, \bar{\omega}_{1 / 2}^{h}, \widetilde{\omega}_{1 / 2}^{h}, \omega_{1 / 2}^{h}, \bar{\omega}^{\tau}, \omega^{\tau}$, respectively, and let $Z$ be one

of the functions $U, \widetilde{U}, \stackrel{\circ}{U}, \bar{V}, \widetilde{V}$, and $V$. In this section $\dot{U} \in R\left(\bar{\omega}^{h}\right)$ is zero continuation function of $U$, and $\stackrel{*}{Y} \in R\left(\stackrel{\circ}{\omega}^{\tau}\right)$ is zero continuation function of 
$\stackrel{\circ}{Y}$, and $\stackrel{*}{U} \in R\left(\bar{\omega}^{h}\right)$ is zero continuation function of $\widetilde{U}$, and $\ddot{V} \in R\left(\bar{\omega}_{1 / 2}^{h}\right)$ is even continuation function $\left(\ddot{V}_{-1 / 2}=V_{1 / 2}, \ddot{V}_{n+1 / 2}=V_{n-1 / 2}\right)$ of $V$.

We introduce the following grid operators. For the functions $Z_{i}=Z\left(\widetilde{q}_{i}\right)$ defined on the grids $\bar{\omega}^{h}$ or $\bar{\omega}_{1 / 2}^{h}$ we assume

$$
\delta Z_{i+1 / 2}=\left(Z_{i+1}-Z_{i}\right) / h_{i+1}, \quad s Z_{i+1 / 2}=\left(Z_{i}+Z_{i+1}\right) / 2, \quad Z \pm_{, i}=Z_{i \pm 1 / 2}
$$

( $i$ is integer or semi-integer indice). Let $\delta^{k}, s^{k}, k=0,1,2$ be powers of operators $\delta, s$. It is not difficult to prove that $\delta\left(Z_{1} Z_{2}\right)=\delta\left(Z_{1}\right) s\left(Z_{2}\right)+s\left(Z_{1}\right) \delta\left(Z_{2}\right)$ and

$$
\begin{array}{r}
|s \bar{V}| \leq 2 s|\bar{V}|, \quad|s U| \leq 2 s|U|, \quad|\delta s U| \leq 2 s|\delta U| \\
|\delta s \bar{V}| \leq K(N) s|\delta \bar{V}|,|\delta s \ddot{V}| \leq K(N) s\left|\bar{\partial}_{t} \dot{\delta} V\right| .
\end{array}
$$

For the functions $Y^{j}=Y\left(t_{j}\right) \in \mathbb{R}\left(\bar{\omega}^{\tau}\right)$ we assume

$$
\begin{gathered}
\stackrel{\vee}{Y^{j}}=Y^{j-1}, 1 \leq j \leq \bar{n}, \quad \widehat{Y}^{k}=Y^{k+1}, 0 \leq k<\bar{n}, \stackrel{\vee}{Y^{0}}=Y^{0}, \bar{\partial}_{t} Y^{0}=0 \\
\bar{\partial}_{t} Y=(Y-\stackrel{\vee}{Y}) / \tau, \quad \partial_{t} Y=(\widehat{Y}-Y) / \tau, \quad s_{t} Y=(Y+\stackrel{Y}{Y}) / 2, \quad I_{\tau}^{0} Y=0, \\
I_{\tau}^{k, j} Y=\sum_{k<l \leq j} Y^{l} \tau_{l}, \stackrel{\circ}{I_{\tau}^{j}} Y=I_{\tau}^{1, j} Y, I^{j} Y=I_{\tau}^{0, j} Y .
\end{gathered}
$$

Then the following formulae are valid:

$$
\begin{gathered}
\bar{\partial}_{t}\left(Y_{1} Y_{2}\right)=\left(\bar{\partial}_{t} Y_{1}\right) Y_{2}+\stackrel{\vee}{Y}_{1}\left(\bar{\partial}_{t} Y_{2}\right), \\
I_{\tau}\left(Y_{1} Y_{2}\right)=Y_{1} I_{\tau} Y_{2}-I_{\tau}\left(\bar{\partial}_{t} Y_{1} \stackrel{\vee}{I}_{\tau} Y_{2}\right), \\
I_{\tau}^{k, j}\left(Y_{1} \bar{\partial}_{t} Y_{2}\right)=Y_{1}^{j} Y_{2}^{j}-Y_{1}^{k} Y_{2}^{k}-I_{\tau}^{k, j}\left(\bar{\partial}_{t} Y_{1} Y_{2}\right) .
\end{gathered}
$$

From the norm $L_{q}(\Omega), \quad L_{q}(\Omega), \quad L_{r}(0, T) \quad$ for $q, r \in[1, \infty)$ we get the norms $\|\cdot\|_{q, \bar{\omega}^{h}},\|\cdot\|_{q, \omega_{1 / 2}^{h}},\|\cdot\|_{r, \omega^{\tau}}$ if trapezoidal, midvalue, right rectangular integration rules are used, respectively. We introduce the following norms ( $\omega$ is one of the grids on $[0, L]$ )

$$
\begin{gathered}
\|U\|_{q, \omega^{h}}=\|\dot{U}\|_{q, \bar{\omega}^{h}},\|\widetilde{U}\|_{q, \omega^{h}}=\|\stackrel{*}{U}\|_{q, \bar{\omega}^{h}},\|Y\|_{\infty, \omega^{\tau}}=\max _{1 \leq j \leq \bar{n}}\left|Y\left(t_{j}\right)\right|, \\
\|Z\|_{\infty}=\max _{x \in \omega}|Z(x)|,\|\cdot\|\left\|_{q, r, \omega \times \omega^{\tau}}=\right\|\|\cdot\|_{q, \omega} \|_{r, \omega^{\tau}}, q, r \in[1, \infty]
\end{gathered}
$$

as well as the inner product $(Z, \bar{Z})_{\omega}$ such that $(Z, Z)_{\omega}=\|\left. Z\right|_{2, \omega} ^{2}$. Then the formulae of the summation by parts

$$
(U, \delta V)_{\omega^{h}}+(\delta U, V)_{\omega_{1 / 2}^{h}}=U_{n} V_{n-1 / 2}-U_{0} V_{1 / 2}
$$




$$
(U, \delta \widetilde{V})_{\widetilde{\omega}^{h}}+(\delta U, \widetilde{V})_{\omega_{1 / 2}^{h}}=U_{n} \widetilde{V}_{n+1 / 2}-U_{0} \widetilde{V}_{1 / 2}
$$

hold. We denote

$$
\begin{gathered}
\|Z\|_{Q^{l}, \omega}=\left(I_{\tau}^{l}\|Z\|_{2, \omega}^{2}\right)^{1 / 2}, \quad\|Z\|_{2, \infty, Q^{l}, \omega}=\max _{0 \leq j \leq l}\left\|Z^{j}\right\|_{2, \omega}, \\
\|Z\|_{C\left(Q^{l}\right), \omega}=\max _{0 \leq j \leq l}\left\|Z^{j}\right\|_{\infty, \omega}, \quad 0<l \leq \bar{n} .
\end{gathered}
$$

Further on we omit subscripts $\omega, \omega^{\tau}$, and $\omega \times \omega^{\tau}$ denoting a domain of grid functions. We use norms

$$
\begin{gathered}
\|Z\|_{V_{2}\left(Q^{l}\right)}=\|Z\|_{2, \infty, Q^{l}}+\|\delta Z\|_{Q^{l}}, \\
\|Z\|_{Q^{l}}^{(2,1)}=\|Z\|_{V_{2}\left(Q^{l}\right)}+\left\|\bar{\partial}_{t} Z\right\|_{Q^{l}}+\|\delta Z\|_{V_{2}\left(Q^{l}\right)} .
\end{gathered}
$$

Denote $\|\cdot\|_{C}=\|\cdot\|_{C(Q)},\|\cdot\|_{V_{2}}=\|\cdot\|_{V_{2}(Q)},\|\cdot\|^{(2,1)}=\|\cdot\|_{Q}^{(2,1)}, Q=Q^{\bar{n}}$, $\|\cdot\|=\|\cdot\|_{2},\|\cdot\|^{(1)}=\|\cdot\|+\|\delta \cdot\|$.

It is not difficult to prove that $\left(0<\varepsilon \leq 1, p, q, q_{0}, q_{1}, r, r_{0}, r_{1} \in[1, \infty]\right)$

$$
\begin{gathered}
\|s U\|_{p} \leq\|U\|_{p},\|s V\|_{p} \leq K(N)\|V\|_{p}, \\
\|Z\|_{\infty} \leq \varepsilon\|\delta Z\|+K_{\varepsilon}(N)\|Z\|_{1}, \\
\|Z\|_{\infty}+\|\delta Z\|_{\infty} \leq \varepsilon\left\|\delta^{2} Z\right\|+K_{\varepsilon}(N)\|Z\|_{1}, \\
\|Z\|_{q} \leq K(N)(\|\delta Z\|+\|Z\|)^{2 / r}\|Z\|^{1-2 / r} \\
|(a, Z)| \leq \varepsilon\|\delta Z\|^{2}+K_{\varepsilon}(N)\left(\left.\|a\|_{q_{1}}^{r_{1}}\|Z\|\right|^{2-r_{1}}+\|a\|_{q_{1}}\|Z\|\right), \\
\left|\left(a Z_{1}, Z\right)\right| \leq \varepsilon\|\delta Z\|^{2}+K_{\varepsilon}(N)\left(\|Z\|^{2}+\|a\|_{q_{1}}^{r_{1}}\left(\|Z\|^{2}+\left\|Z_{1}\right\|_{\infty}^{2}\right)\right), \\
\left(a Z_{1}, Z\right) \mid \leq \varepsilon\|\delta Z\|^{2}+\varepsilon\left\|\delta Z_{1}\right\|^{2}+K_{\varepsilon}(N)\left(\left(1+\|a\|_{q_{0}}^{r_{0}}\right)\left(\|Z\|^{2}+\left\|Z_{1}\right\|^{2}\right)\right), \\
(2 q)^{-1}+r^{-1}=1 / 4,\left(2 q_{0}\right)^{-1}+r_{0}^{-1}=1,\left(2 q_{1}\right)^{-1}+r_{1}^{-1}=5 / 4 .
\end{gathered}
$$

Lemma 2.1 (Difference Gronwall's L., see [1,3]). Let functions $A^{(1)}, A^{(2)}$, $A^{(3)}, B^{(1)}, B^{(2)}, F$ be defined on the grid $\omega^{\tau}$. If function $Y \geq 0$ is defined on the grid $\bar{\omega}^{\tau}$ and satisfies inequality

$$
Y \leq \bar{Y}^{0}+I_{\tau}\left(A^{(1)} Y+A^{(2)} \stackrel{\vee}{Y}+B^{(1)} Y^{1 / 2}+B^{(2)} \stackrel{\vee}{Y}{ }^{1 / 2}+F\right)+\stackrel{\vee}{I_{\tau}}\left(A^{(3)} Y\right)
$$

with $\bar{Y}^{0}=$ const $\geq Y^{0} \geq 0$ and $\tau_{j} A^{(1), j} \leq 1 / 2,1 \leq j \leq \bar{n}$, then the estimate

$$
\|Y\|_{\infty}^{1 / 2} \leq\left(\left(\bar{Y}^{0}+\|F\|_{1}\right)^{1 / 2}+\|B\|_{1} \exp \left(\|A\|_{1}\right)\right) \exp \left(\|A\|_{1}\right)
$$

is valid. There $A=\left|A^{(1)}\right|+\left|A^{(2)}\right|+\left|A^{(3)}\right|+,\left.A^{(3)}\right|_{j=\bar{n}}=0, B=\left|B^{(1)}\right|+$ $\left|B^{(2)}\right|,\|\cdot\| r=\|\cdot\| r, w^{\tau}$. 


\section{SPECIAL LINEAR PARABOLIC EQUATION}

Now we consider another linear difference scheme (LDS1) [4]:

$$
\bar{\partial}_{t}(\alpha V)=\beta \delta \Pi+\Phi,\left.\quad V\right|_{i=0}=\left.\Pi\right|_{i=n+1 / 2}=0,\left.\quad V\right|^{j=0}=V^{0} .
$$

The unknown function $V$ is defined on the grid $\bar{\omega}^{h} \times \bar{\omega}^{\tau}$ and $\left.V^{0}\right|_{i=0}=0, \Pi$ is defined on the grid $\widetilde{\omega}_{1 / 2}^{h} \times \omega^{\tau}$. If we suppose that $P^{0}=P^{1}, \gamma^{0}=\gamma^{1}, \kappa^{0}=\kappa^{1}$ then $\Pi=\Pi(V)=\Pi(V, \kappa, \gamma, P)=\kappa \delta V+\gamma s V-P$ is defined on the grid $\omega_{1 / 2}^{h} \times \bar{\omega}^{\tau}$. Eq. (4) is defined on the grid $\widetilde{\omega}^{h} \times \omega^{\tau}$. We suppose $\left.\Phi_{c}\right|_{i=n+1 / 2}=0$. Let $L=L(Z)=L(Z, \beta, \kappa, \gamma, P)=\beta \delta \Pi, \Lambda=\Lambda(Z)=\Lambda(Z, \beta, \kappa, \gamma, P, \Phi)=$ $L(Z)+\Phi$. We denote $\Pi_{l}(V)=\Pi\left(V, \kappa_{l}, \gamma_{l}, P_{l}\right), \Lambda_{l}(Z)=\Lambda_{l}\left(Z, \beta_{l}, \kappa_{l}, \gamma_{l}, P_{l}, \Phi_{l}\right)$, $l=1,2$; and $\vec{\kappa}=\left(\kappa_{1}, \kappa_{2}\right), \vec{\gamma}=\left(\gamma_{1}, \gamma_{2}\right), \vec{P}=\left(P_{1}, P_{2}\right), \vec{\Phi}=\left(\Phi_{1}, \Phi_{2}\right)$.

Lemma 3.1. Let $N^{-1} \leq \kappa_{1}, \kappa_{2} \leq N$, and $q_{l} \in[1, \infty], r_{l} \in[1,2], l=$ $1,2,3,4$, and $\left(2 q_{l}\right)^{-1}+r_{l}^{-1}=1$.

a) Then

$$
\begin{aligned}
& K(N)^{-1}|| \delta V\left\|^{2} \leq\left(\Pi_{1}, \Pi_{2}\right)+K(N)\right\| \vec{P}\left\|^{2}+K(N)\left(1+\|\vec{\gamma}\|_{2 q_{1}}^{2 r_{1}}\right)\right\| V \|^{2} \\
& \left(\Pi_{1}, \Pi_{2}\right) \leq K(N)\|\delta V\|^{2}+K(N)\|\vec{P}\|^{2}+K(N)\left(1+\|\vec{\gamma}\|_{2 q_{1}}^{2 r_{1}}\right)\|V\|^{2} .
\end{aligned}
$$

b) If $N^{-1} \leq \beta_{1}, \beta_{2} \leq N$ then

$$
\begin{aligned}
K(N)^{-1}\left\|\delta^{2} V\right\|^{2} & \leq\left(\Lambda_{1}, \Lambda_{2}\right)+K(N) d_{*}^{2} \\
\left(\Lambda_{1}, \Lambda_{2}\right) & \leq K(N)\left(\left\|\delta^{2} V\right\|^{2}+d_{*}^{2}\right)
\end{aligned}
$$

where

$$
\begin{aligned}
d_{*}^{2}= & \left(\|\vec{P}\|^{(1)}\right)^{2}+\|\vec{\Phi}\|^{2}+\left(1+\|\delta \vec{\gamma}\|_{2 q_{2}}^{2 r_{2}}\right)|| V \|^{2} \\
& +\left(1+\|\vec{\gamma}\|_{2 q_{3}}^{2 r_{3}}+\|\delta \vec{\kappa}\|_{2 q_{4}}^{2 r_{4}}\right)\|\delta V\|^{2} .
\end{aligned}
$$

P r o o f. a) As $\Pi=\kappa \delta V+\gamma s V-P$ and $\|\gamma s V-P\|^{2} \leq K\|P\|^{2}+$ $K\left(|\gamma|^{2}, s V s V\right) \leq \varepsilon\|\delta V\|^{2}+K\|P\|^{2}+K\left(1+\|\gamma\|_{2 q}^{2 r}\right)\|V\|^{2}$ then we can consider only the case $\gamma_{1}=\gamma_{2}=0$. In this case we have

$$
\begin{array}{r}
K^{-1}|| \delta V\left\|^{2} \leq\left(\kappa_{1} \delta V, \kappa_{2} \delta V\right) \leq K\right\| \delta V\left\|^{2}, \quad\left|\left(-P_{1},-P_{2}\right)\right| \leq K\right\| \vec{P} \|^{2} \\
\left|\left(\kappa_{i} \delta V,-P_{j}\right)\right| \leq \varepsilon\|\delta V\|^{2}+K\|\vec{P}\|^{2}, i, j=1,2, i \neq j .
\end{array}
$$

So the part a) is proved.

b) As $\|\gamma s V-P\|^{2} \leq \varepsilon\left\|\delta^{2} V\right\|^{2}+K\|P\|^{2}+K\left(1+\|\gamma\|_{2 q}^{2 r}\right)\|V\|^{2}$ and

$$
\begin{aligned}
& \|\delta \gamma s s V+s \gamma \delta s V-\delta P\|^{2} \\
& \left.\leq \varepsilon\left\|\delta^{2} V\right\|^{2}+K\left(1+\|\delta \gamma\|_{2 q}^{2 r}\right)\right)\|V\|^{2}+K\left(1+\|\gamma\|_{2 q}^{2 r}\right)\|\delta V\|+K\|\delta P\|^{2}
\end{aligned}
$$


then we can consider only the case $\gamma_{1}=\gamma_{2}=0$. We have $A=\beta s \kappa \delta^{2} V+$ $\beta \delta \kappa s \delta V-\beta \delta P+\Phi$ and

$$
\begin{aligned}
& \|\beta \delta \kappa s \delta V-\beta \delta P+\Phi\|^{2} \\
& \leq \varepsilon\left\|\delta^{2} V\right\|^{2}+K\left(\|\Phi\|^{2}+\|\delta P\|^{2}+\left(1+\|\delta \kappa\|_{2 q}^{2 r}\right)\|\delta V\|^{2}\right) .
\end{aligned}
$$

So we must consider only case $A=\beta \delta^{2} V+\Phi$. Then the proof of case b) follows from inequalities

$$
\begin{aligned}
K^{-1}\left\|\delta^{2} V\right\|^{2} & \leq\left(\beta_{1} \delta^{2} V, \beta_{2} \delta^{2} V\right) \leq K\left\|\delta^{2} V\right\|^{2},\left|\left(\Phi_{1}, \Phi_{2}\right)\right| \leq K\|\vec{\Phi}\|^{2} \\
& \left|\left(\beta_{i} \delta^{2} V, \Phi_{j}\right)\right| \leq \varepsilon\left\|\delta^{2} V\right\|^{2}+K\left\|\vec{\Phi}_{j}\right\|^{2}, i, j=1,2, i \neq j
\end{aligned}
$$

We consider equation (4) and denote $\|Z\|_{Q}^{(-1)}=\|\| Z\left\|^{(-1) ; 1}\right\|_{2, \omega^{\tau}}$, $Z^{-}=\min (Z, 0)$.

LEMMA 3.2. Let $N^{-1} \leq \kappa, \beta \leq N, N^{-1} \leq \alpha$, and $q_{l}, r_{l} \in[1, \infty],\left(2 q_{l}\right)^{-1}+$ $r_{l}^{-1} \leq 1, l=1, \ldots, 6,\left(2 q_{l}\right)^{-1}+r_{l}^{-1} \leq 5 / 4, l=7,8$.

a) If $\left\|\alpha^{0}\right\|_{\infty} \leq N,\left\|\left(\bar{\partial}_{t} \alpha\right)^{-}\right\|_{q_{1}, r_{1}} \leq N,\|\gamma\|_{2 q_{2}, 2 r_{2}} \leq N,\|\delta \beta\|_{2 q_{3}, 2 r_{3}} \leq N$ and $\tau_{\max } \leq \tau^{0}(N)$, then

$$
\begin{aligned}
& \left\|\sqrt{\tau} \bar{\partial}_{t} V\right\|_{Q}^{2}+\|V\|_{V_{2}}+\|\Pi\|_{Q} \leq K(N)\left(\left\|V^{0}\right\|+\left\|\Phi_{a}^{0}\right\|\right. \\
& \left.+\left\|\tau^{-1 / 2} \Phi_{a}\right\|_{Q}+\left\|\Phi_{b}\right\|_{q_{7}, r_{7}}+\left\|\Phi_{c}\right\|_{Q}+\|P\|_{Q}+\|V\|_{Q}^{2}\right) ;
\end{aligned}
$$

b) If $\alpha \leq N,\left\|\bar{\partial}_{t} \alpha\right\|_{Q} \leq N,\left\|\partial_{t} \kappa\right\|_{q_{4}, r_{4}} \leq N,\|\delta \kappa\|_{2 q_{5}, 2 r_{5}} \leq N,\|\gamma\|_{2, \infty} \leq N$, $\|\delta \gamma\|_{2 q_{6}, 2 r_{6}} \leq N,\left\|\partial_{t} \gamma\right\|_{q_{8}, r_{8}} \leq N$ and $\tau_{\max } \leq \tau^{0}(N)$, then

$$
\begin{aligned}
\|V\|^{(2,1)}+ & \left\|\sqrt{\tau \bar{\partial}_{t}} \delta V\right\|_{Q}+\|\delta \Pi\|_{Q} \leq K(N)\left(\left.\left\|V^{0}\right\|\right|^{(1)}+\|\Phi\|_{Q}\right. \\
& \left.+\|P\|_{V_{2}(Q)}+\left\|\bar{\partial}_{t} P\right\|_{Q}^{(-1)}+\left\|\sqrt{\tau} \bar{\partial}_{t} P\right\|_{Q}+\|V\| V_{V_{2}}\right)
\end{aligned}
$$

If $q_{1}=q_{2}=q_{3}=1, r_{1}=r_{2}=r_{3}=\infty$ in the case a) or $q_{5}=1, r_{5}=\infty$ in the case b) then we can omit condition $\tau_{\max } \leq \tau^{0}(N)$.

$\mathrm{P}$ r o o f. In this proof let conditions for $q_{l}, r_{l}$ be equalities because $\|\cdot\|_{q, r} \leq$ $K\|\cdot\|_{\bar{q}, \bar{r}}$ if $q \leq \bar{q}$ and $r \leq \bar{r}$.

a) Let $d=\left\|V^{0}\right\|+\left\|\overline{\Phi_{a}^{0}}\right\|+\left\|\tau^{-1 / 2} \Phi_{a}\right\|_{Q}+\left\|\Phi_{b}\right\|_{q_{1}, r_{1}}+\left\|\Phi_{c}\right\|_{Q}+\|P\|_{Q}$. We take the inner product of equation (4) with $V$, apply operator $I_{\tau}^{l}$ (we omit index $l$ below in the proof) and get

$$
\begin{aligned}
& \left(\bar{\partial}_{t} \alpha, V^{2}\right)_{Q}+0.5\left\|\sqrt{\tau}{ }^{\vee}{ }^{1 / 2} \bar{\partial}_{t} V\right\|_{Q}^{2}+0.5\|\sqrt{\alpha} V\|^{2} \\
& =0.5\left\|\sqrt{\alpha^{0}} V^{0}\right\|^{2}+(\delta \Pi, \beta V)_{Q}+\left(\Phi_{b}, V\right)_{Q}+\left(\delta \Phi_{c}, V\right)_{Q} \\
& \quad+\left(\Phi_{a}, V\right)-\left(\Phi_{a}^{0}, V^{0}\right)-\left(\tau^{-1 / 2} \stackrel{\vee}{\left.\Phi_{a}, \sqrt{\tau} \bar{\partial}_{t} V\right)_{Q} .}\right.
\end{aligned}
$$


If $\bar{\partial}_{t} \alpha \geq 0$ we bound the first summand by zero, else we estimate $\left(-\bar{\partial}_{t} \alpha, V^{2}\right)_{Q}$ $\leq \varepsilon\|\delta V\|_{Q}^{2}+K I_{\tau}\left(\left(1+\left.\left\|\left(\bar{\partial}_{t} \alpha\right)^{-}\right\|\right|_{q_{1}} ^{r_{1}}\right)\|V\|^{2}\right)$. Using the formula of summation by parts we get $\left(\delta \Phi_{c}, V\right)_{Q}=-\left(\Phi_{c}, \delta V\right)_{Q}$ and

$$
(\delta \Pi, \beta V)_{Q}=-(\Pi, \delta \beta s V)_{Q}-(\kappa s \beta \delta V, \delta V)_{Q}+(s \beta P, \delta V)_{Q}-(\gamma s V, \delta V)_{Q} .
$$

We estimate

$$
\begin{aligned}
& \left|(\Pi, \delta \beta s V)_{Q}\right| \leq \varepsilon\|\Pi\|_{Q}^{2}+\varepsilon\|\delta V\|_{Q}^{2}+K I_{\tau}\left(\left(1+\|\delta \beta\|_{2 q_{3}}^{2 r_{3}}\right)|| V \|^{2}\right), \\
& \left|(\gamma s V, \delta V)_{Q}\right| \leq \varepsilon\|\delta V\|_{Q}^{2}+K I_{\tau}\left(\left(1+\|\gamma\|_{2 q_{2}}^{2 r_{2}}\right)|| V \|^{2}\right), \\
& \left|\left(\Phi_{b}, V\right)_{Q}\right| \leq \varepsilon\|\delta V\|_{Q}^{2}+\varepsilon\|V\|^{2}+K \stackrel{\vee}{I}_{\tau}\left(\left(\left\|\Phi_{b}\right\|_{q_{7}}^{r_{7}}+\left\|\Phi_{b}\right\|_{q_{7}}\right)\|V\|\right)+K d^{2} .
\end{aligned}
$$

From lemma 3.1a and obtained estimates we get the inequality

$$
\begin{array}{r}
\|V\|^{2} \leq K I_{\tau}\left(\left(\left.\left\|\left(\bar{\partial}_{t} \alpha\right)^{-}\right\|\right|_{q_{1}} ^{r_{1}}+\|\gamma\|_{2 q_{2}}^{2 r_{2}}+\|\delta \beta\|_{2 q_{3}}^{2 r_{3}}\right)|| V \|^{2}\right) \\
+K \stackrel{\vee}{I}_{\tau}\left(\left(\left\|\Phi_{b}\right\|_{q_{7}}^{r_{7}}+\left\|\Phi_{b}\right\|_{q_{7}}\right)\|V\|\right)+K d^{2} .
\end{array}
$$

Now we use Gronwall's lemma which proves this part of the lemma. If $\left\|\left(\bar{\partial}_{t} \alpha\right)^{-}\right\|_{1, \infty}+\|\gamma\|_{2, \infty}+\|\delta \beta\|_{2, \infty} \leq N$ then $I_{\tau}\left((\cdot)\|V\|^{2}\right) \leq K d^{2}$.

b) Let $d=\left.\left\|V^{0}\right\|\right|^{(1)}+\|\Phi\|_{Q}+\|P\|_{V_{2}(Q)}+\left\|\bar{\partial}_{t} P\right\|_{Q}^{(-1)}+\left\|\sqrt{\tau} \bar{\partial}_{t} P\right\|_{Q}+\|V\|_{V_{2}}$. We consider the case $\alpha=1$ because the general case we get with $\Phi^{\prime}=$ $\Phi_{\alpha}^{\vee-1}-\bar{\partial}_{t} \alpha \alpha^{-1} V$. We take inner product of equation (4) with $\left(\beta^{-1} \bar{\partial}_{t} V-\delta \Pi\right) / 2$, apply the operator $I_{\tau}^{l}$ which yields

$$
K^{-1}\left(\left\|\bar{\partial}_{t} V\right\|_{Q}^{2}+\|\delta \Pi\|_{Q}^{2}\right) \leq\left(\bar{\partial}_{t} V, \delta \Pi\right)_{Q}+K d^{2} .
$$

Using the formula of summation by parts we have

$$
\left(\bar{\partial}_{t} V, \delta \Pi\right)_{Q}=-\left(\bar{\partial}_{t} \delta V, \kappa \delta V\right)_{Q}+\left(\bar{\partial}_{t} \delta V, P\right)_{Q}+\left(\bar{\partial}_{t} \delta V, \gamma s V\right)_{Q}
$$

Each summand on the righthand side of this equality we rewrite in the form

$$
\begin{array}{r}
-0.5\left(\kappa, \tau\left(\bar{\partial}_{t} \delta V\right)^{2}\right)_{Q}-0.5\|\sqrt{\kappa} \delta V\|^{2}+0.5\left\|\sqrt{\kappa^{0}} \delta V^{0}\right\|^{2}+0.5 \stackrel{\vee}{I_{\tau}}\left(\partial_{t} \kappa,|\delta V|^{2}\right), \\
(P, \delta V)-\left(P^{0}, \delta V^{0}\right)-I_{\tau}\left(\bar{\partial}_{t} P, \delta V\right)+I_{\tau}\left(\tau \bar{\partial}_{t} P, \bar{\partial}_{t} \delta V\right), \\
-(\delta V, \gamma s V)+\left(\delta V^{0}, \gamma^{0} s V^{0}\right)+\stackrel{\vee}{I}_{\tau}\left(\partial_{t} \gamma, s V \delta V\right)+I_{\tau}\left(s \bar{\partial}_{t} V, \gamma \delta V\right),
\end{array}
$$

and estimate the inner products

$$
\left|\stackrel{\vee}{I_{\tau}}\left(\partial_{t} \kappa,|\delta V|^{2}\right)\right| \leq \varepsilon|| \delta^{2} V \|_{Q}^{2}+K \stackrel{\vee}{I_{\tau}}\left(\left\|\partial_{t} \kappa\right\|_{q_{4}}^{r_{4}}\|\delta V\|^{2}\right)+K d^{2}
$$




$$
\begin{array}{r}
\left|\left(\bar{\partial}_{t} \delta V, P\right)_{Q}\right| \leq \varepsilon\|\delta V\|^{2}+\varepsilon\left\|\sqrt{\tau \partial}_{t} \delta V\right\|_{Q}^{2}+K d^{2}, \\
\left|\vee I_{\tau}\left(\partial_{t} \gamma, s V \delta V\right)\right| \leq \varepsilon|| \delta^{2} V \|_{Q}+K \stackrel{\vee}{I}_{\tau}\left(\left\|\partial_{t} \gamma\right\|_{q_{8}}^{r_{8}}\|\delta V\|^{2}\right)+K d^{2}, \\
\left|I_{\tau}\left(s \bar{\partial}_{t} V, \gamma \delta V\right)\right| \leq \varepsilon\left\|\bar{\partial}_{t} V\right\|^{2}+K I_{\tau}\|\delta V\|^{2}+K d^{2} .
\end{array}
$$

From lemma $3.1 \mathrm{~b}$ and obtained estimates we get the inequality

$$
\begin{array}{r}
\|\delta V\|_{Q}^{2} \leq K I_{\tau}\left(\|\delta \stackrel{\vee}{V}\|^{2}+\|\delta \kappa\|_{2 q_{5}}^{2 r_{5}}\|\delta V\|^{2}\right) \\
+K \stackrel{\vee}{I}_{\tau}\left(\left(\left\|\partial_{t} \kappa\right\|_{q_{4}}^{r_{4}}+\left\|\partial_{t} \gamma\right\|_{q_{8}}^{r_{8}}\right)\|\delta V\|^{2}\right)+K d^{2} .
\end{array}
$$

Now we use Gronwall's lemma which proves this part of the lemma.

At the time this reaseach was provided, the author was employed at the Institute of Mathematics and Informatics, Vilnius Gediminas Technical University, and Kaunas Vytautas the Great University.

\section{REFERENCES}

[1] A.A. Amosov and A.A. Zlotnik, Difference schemes of the second order of accuracy for equations of the one-dimensional motion of a viscous gas,in: Zh. Vychisl. Mat. i Mat. Fiz.,, 27(1987) 1032-10494;

[2] A.A. Amosov and A.A. Zlotnik, A family of difference schemes for equations of onedimensional magnetogasdynamics: properties and error estimates "in the large",in: Soviet Math. Dokl., 37(1988) 545-549;

[3] A.A. Amosov and A.A. Zlotnik, A difference scheme on a nonuniform grid for equations of one-dimensional magnetogasdynamics, in: Zh. Vychisl. Mat. i Mat. Fiz., 29(1989) 521-534(in Russian).

[4] A. S̆tikonas, Some estimates for the difference scheme of the linear parabolic equation in: Thesis of XXXIV Conference of Lith. Math. Soc., Vilnius(1993) 39-40. 